\title{
Comparação da motivação para a prática esportiva de jovens atletas de atletismo em razão do sexo, tempo de prática e grupo de competição
}

\author{
Comparison of motivation to practice sports in young athletes based on sex, \\ practice time and competition group
}

Giovana Correia da Silva', Luciana Boligon Refundini', Daniel Vicentini de Oliveira², Paula Caroline Teixeira Marroni , José Roberto Andrade do Nascimento Júnior ${ }^{3}$, Ana Luiza Barbosa Anversa ${ }^{1}$

1 Universidade Estadual de Maringá (UEM), Maringá, Brasil

2 Universidade Cesumar (UNICESUMAR), Maringá, Brasil

${ }^{3}$ Universidade Federal do Vale do São Francisco (UNIVASF), Petrolina, Brasil

\section{HISTÓRICO DO ARTIGO \\ Recebido: 29 setembro 2020

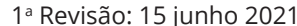 \\ 2a Revisão: 8 fevereiro 2022 \\ Aprovado: 11 fevereiro 2022}

\section{PALAVRAS-CHAVE:}

Esporte; Motivação;

Atletismo.

\section{KEYWORDS:}

Sport; Motivation;

Athletics.

\section{PUBLICADO:}

22 fevereiro 2022

\section{RESUMO}

OBJETIVO: Comparar a motivação para a prática esportiva de jovens atletas do atletismo em função do sexo, tempo de prática e grupo de competição.

MÉTODOS: Estudo transversal, realizado com 30 alunos/atletas, sendo 19 do sexo masculino e 11 do sexo feminino, das categorias de provas de pista e campo, entre 9 e 17 anos. Foi utilizada a Escala de Motivação para o Esporte II (SMS II). Para análise dos dados foi utilizado os testes Shapiro-Wilk, Kruskal-Wallis e U de Mann-Whitney $(p<0,05)$.

RESULTADOS: Os resultados demonstraram que os atletas são mais motivados por fatores intrínsecos $(\mathrm{Md}=7,00)$. Em relação ao sexo, as atletas femininas tiveram uma diferença significativa na regulação externa comparada aos atletas masculinos $(p=0,04)$ e os atletas de provas de campo demonstraram mais motivados extrinsecamente do que os atletas das provas de pista $(p=0,03)$.

CONCLUSÃO: Concluiu-se que o sexo e o tipo de prova são fatores intervenientes na motivação extrínseca e desmotivação dos atletas de atletismo.

\section{ABSTRACT}

OBJECTIVE: To compare the motivation for the sports practice of young athletes in terms of sex, practice time and competition group.

METHODS: Cross-sectional study, carried out with 30 students / athletes, 19 of whom were male and 11 females, from the categories of track and field events, between 9 and 17 years old. The Sport Motivation Scale II (SMS II) was used. For data analysis, the Shapiro-Wilk, Kruskal-Wallis and U Mann-Whitney tests were used $(\mathrm{p}<0.05)$.

RESULTS: The results showed that athletes are more motivated by intrinsic factors $(\mathrm{Md}=7.00)$. In relation to sex, female athletes had a significant difference in external regulation compared to male athletes $(p=0.04)$ and athletes in field events showed more extrinsically motivated than athletes in track events $(p=0.03)$.

CONCLUSION: It was concluded that gender and type of race are intervening factors in the extrinsic motivation and demotivation of track and field athletes. 
2 SILVA et al.

Comparação da motivação para a prática esportiva de jovens atletas de atletismo em razão do sexo, tempo de prática e grupo de competição

\section{INTRODUÇÃO}

O atletismo se configurou como modalidade competitiva nos Jogos Olímpicos em Olímpia, na Grécia, em 776 a.C., tendo seus primeiros registros competitivos no Brasil no ano de 1910 (CONFEDERAÇÃO BRASILEIRA DE ATLETISMO, 2021). O interesse pela prática competitiva desse esporte vem aumentando nos últimos anos, por ser uma modalidade que atende demandas motoras de um vasto leque de atividades humanas (ANDRADE; COUTINHO, 2019).

Além disso, a prática do atletismo oferece ao praticante diferentes grupos de competição como: 1) provas de pista: corridas rasas (velocidades, meio-fundo e fundo), com barreiras, com obstáculos e revezamento; 2) provas de campo: saltos (salto em distância, salto triplo, salto em altura e salto com vara), arremessos de peso, lançamentos (lançamento do dardo, lançamento do disco e lançamento do martelo); 3) provas combinadas (decatlo para homens e heptatlo para mulheres, que agregam provas de pista e de campo); 4) corridas de rua (maratona e revezamento); 5) corridas em campo (cross country); e 6) marcha atlética (CONFEDERAÇÃO BRASILEIRA DE ATLETISMO, 2021). Cada grupo possui suas especificidades, o que acaba por demandar diferentes habilidades e motivações de seu praticante, por isso faz-se necessário conhecer o que motiva o praticante para a escolha da modalidade/prova.

A motivação é algo individual, por isso não conseguimos motivar uma pessoa, mas sim propiciar meios de estimular e/ou incentivar, e esses meios resultaram em um quadro motivacional intrínseco ou extrínseco. Frente a isso, a motivação pode ser definida como um processo ativo, proposital e dirigido a uma meta, na qual depende de fatores internos (motivação intrínseca: pessoais, prazer, satisfação) e externos (motivação extrínseca: ambientais, recompensas, prêmios) (SAMULSKI, 2002).

Uma das teorias que abordam a motivação é a Teoria da Autodeterminação indicando que a motivação se dá por meio da satisfação de necessidades psicológicas básicas de competência e autonomia, deste modo a motivação é um contínuo que perpassa da desmotivação, para a motivação extrínseca e diferentes níveis de regulação (externa, passeada em recompensas; introjetada, envolvendo o ego e autocontrole; identificada, denotando importância pessoal e valorização consciente e; integrada, que estabelece metas) até se chegar a motivação intrínseca, sendo assim, o indivíduo se sentirá motivado a medida que compreende os motivos intrínsecos e extrínsecos que induzem o mesmo a aderir ou não às práticas de exercícios físicos (DECY; RYAN, 1985; DECY; RYAN, 2009).

No cenário esportivo há duas fontes motivacionais relacionadas que influenciam o desempenho e a participação na modalidade. A motivação para a realização, que indica o domínio da tarefa, o desempenho para alcançar excelência e superar obstáculo, fazendo com que outros se orgulhem do seu talento; e, para a competitividade que é a disposição para lutar por satisfação ao ter seu padrão de excelência comparado com de outros, na presença de avaliadores, atingindo-se metas ou premiações ao superar o outro (WEINBERG; GOULD, 2016).

Estudos realizados por Bento et al. (2008), Souza et al. (2016) e Amaral, Silva e Miranda (2017) verificaram que a motivação para o treinamento do atletismo está mais rela- cionada a fatores intrínsecos como a autossuperação, realização de um sonho ao participar de uma prova ou evento específico como uma maratona, o prazer, a competência técnica, a afiliação geral e a forma física, e menos a fatores extrínsecos como emoções e afiliação específica. Conhecer a fonte motivacional, em especial em relação ao sexo e ao tempo de prática, pode vir a favorecer a intervenção do profissional de educação física, uma vez que conforme indicado por Da Silva et al. (2019) fatores como idade, gênero e tempo de prática pode vir a influenciar a motivação e ou permanência na prática da corrida.

Mesmo frente a tais achados, nota-se que poucos são os estudos que tratam da temática e que buscam investigar as características motivacionais dos atletas por estilo de prova. Diante do exposto, a presente pesquisa teve como objetivo comparar a motivação para a prática esportiva de jovens atletas do atletismo em função do sexo, tempo de prática e grupo de competição.

\section{MÉTODOS}

A presente pesquisa, caracteriza-se como um estudo quantitativo, observacional e transversal, aprovada no Comitê de Ética em Pesquisa (CEP) da União de Faculdades Metropolitanas de Maringá (UNIFAMMA), sob o Parecer $n^{\circ}$. 2.208.016 (CAAE: 70947617.3.0000.8036).

A pesquisa foi realizada junto a atletas da modalidade de atletismo, vinculados a categorias de base de dois centros de treinamento, da cidade de Maringá, Paraná. A amostra foi composta por 30 alunos/atletas, sendo 19 do sexo masculino e 11 do sexo feminino das categorias de provas de pista e campo, entre 9 e 17 anos (13,6 63,7 anos). Vale ressaltar, que nessa margem etária, se tem atletas da categoria pré-mirim (até 12 anos), mirim (até 14 anos) e menores (até 17 anos) e juvenil (até 19 anos).

Foram considerados os seguintes critérios de inclusão: treinar há mais de três meses a modalidade de atletismo; ter participado de pelo menos uma competição regional; concordar em participar da pesquisa e trazer o Termo de Consentimento Livre e Esclarecido (TCLE) assinado pelos pais ou responsável.

Foi utilizado um questionário sociodemográfico elaborado pelos próprios autores com questões referentes ao sexo, idade, tempo de prática, tipo de competição e categoria. O instrumento utilizado para a coleta de dados foi a "Escala de Motivação para o Esporte II (SMS II)", que foi desenvolvida por Brière, Vallerand, Blais e Pelletier (1995) e validada na versão brasileira por Nascimento Junior et al. (2014). O questionário contém 18 questões, em Escala Likert de sete pontos, num continuum de "não corresponde nada" (1) a "corresponde exatamente" (7), distribuídas em seis subescalas sendo: regulação intrínseca, regulação integrada, regulação identificada, regulação introjetada, regulação externa, desmotivação.

O convite para a participação dos atletas ocorreu em horário de treino e junto aos professores/técnicos foi acordada a data para coleta de dados. Na data estipulada, no próprio local de treino antes ou após as sessões de treinamento, os pesquisadores explicaram os objetivos da pesquisa e instrumento e, mediante o documento de autorização, o SMS II foi aplicado individualmente, juntamen- 
te com um questionário sociodemográfico para se traçar sexo, tempo de treino e tipo de prova.

Para análise dos dados foi utilizada estatística descritiva e inferencial com apresentação dos dados em mediana e intervalo interquartílico em função da não normalidade dos dados por meio do teste de Shapiro-Wilk. Para a análise os dados foram agrupados em categorias. Na variável tempo de prática a amostra foi dividida em praticantes com tempo de prática menor que um ano, de um ano a três anos e com mais de três anos na modalidade, com 10 atletas em cada grupo.

Já em relação às provas, as respostas foram divididas de acordo com os grupos de competição do atletismo, sendo provas de pista (corridas rasas, corridas com barreiras ou com obstáculos) com 23 atletas e provas de campo (saltos, arremesso e lançamentos) com sete atletas. Para a comparação dos estilos regulatórios da motivação em relação ao tempo de prática foi utilizado o teste de Kruskal-Wallis. Na comparação em relação ao sexo e grupo de competição foi utilizado o teste de U de Mann-Whitney. A significância adotada foi de $p<0,05$. Todas as análises foram conduzidas no software SPSS versão 25.0.

\section{RESULTADOS E DISCUSSÃO}

No primeiro momento foi realizada uma análise descritiva dos estilos regulatórios da motivação de praticantes de atletismo (Tabela 1).

Tabela 1. Análise descritiva dos estilos regulatórios da motivação de praticantes de atletismo de Maringá, Paraná (2019).

\begin{tabular}{lc}
\hline Estilos Regulatórios & Md (Q1; Q3) \\
\hline Regulação Intrínseca & $7,00(7,00 ; 7,00)$ \\
Regulação Integrada & $6,84(6,67 ; 7,00)$ \\
Regulação Identificada & $7,00(6,00 ; 7,00)$ \\
Regulação Introjetada & $7,00(7,00 ; 7,00)$ \\
Regulação Externa & $3,33(2,30 ; 5,00)$ \\
Desmotivação & $1,32(1,00 ; 2,60)$ \\
\hline
\end{tabular}

Constata-se que os estilos regulatórios de motivação dos atletas que fizeram parte da amostra apresentam maiores índices de motivação regulatória intrínseca, identificada e introjetada. Verificou-se também que o menor índice regulatório se deu pela desmotivação, o que pode vir a ser um fator positivo no rendimento esportivo dos mesmos.

Ao avaliar o constructo da motivação, na Teoria da Autodeterminação, constatou-se que não houve diferença significativa na variável tempo de prática $(p \geq 0,05)$. Ao comparar os estilos regulatórios da motivação em função ao sexo (Tabela 2 ) houve diferenças significativas $(p=0,04)$ apenas na variável "Regulação Externa", na qual as atletas do sexo feminino apresentaram maiores índices quando comparadas aos do sexo masculino.

Por fim, ao comparar os estilos regulatórios da motivação em função do grupo de competição (provas de pista e provas de campo) (Tabela 3), houve diferenças significativas na variável "Regulação Externa" $(p=0,03)$. Verificou-se que os atletas das provas de campo apresentaram maiores índices de regulação externa quando comparadas aos de pista.

Tabela 2. Comparação dos estilos regulatórios da motivação em função do sexo de praticantes de atletismo de Maringá, Paraná (2019).

\begin{tabular}{lccc}
\hline \multirow{2}{*}{ Estilos Regulatórios } & $\begin{array}{c}\text { Masculino } \\
(\mathrm{n}=19)\end{array}$ & $\begin{array}{c}\text { Feminino } \\
(\mathrm{n}=11)\end{array}$ & \\
\cline { 2 - 3 } & Md (Q1; Q3) & Md $(\mathrm{Q} 1 ; \mathrm{Q} 3)$ & $\mathrm{p}$ \\
\hline Regulação Intrínseca & $7,00(7,00 ; 7,00)$ & $7,00(6,83 ; 7,00)$ & 0,64 \\
Regulação Integrada & $6,67(6,67 ; 7,00)$ & $7,00(6,50 ; 7,00)$ & 1,00 \\
Regulação Identificada & $6,67(6,00 ; 7,00)$ & $7,00(6,66 ; 7,00)$ & 0,37 \\
Regulação Introjetada & $7,00(6,67 ; 7,00)$ & $7,00(6,67 ; 7,00)$ & 0,28 \\
Regulação Externa & $3,00(1,66 ; 4,50)$ & $4,33(3,50 ; 5,00)$ & $0,04 *$ \\
Desmotivação & $1,33(1,00 ; 2,67)$ & $1,33(1,00 ; 2,00)$ & 0,55 \\
\hline
\end{tabular}

* Probabilidade estimada pelo teste de $U$ de Mann-Whitney.

Ao analisar os estilos regulatórios da motivação dos praticantes de atletismo, o fato da motivação regulatória intrínseca, identificada e introjetada apresentarem maiores índices pode-se indicar que, de certo modo, os estilos regulatórios internos atuam como principais fatores motivacionais. Já o menor índice regulatório ser a desmotivação, o que sugere um fator positivo no rendimento esportivo dos mesmos, já que demonstra que os atletas dão sentido (intrínseco e/ou extrínseco) para permanecerem na prática.

Tabela 3. Comparação dos estilos regulatórios da motivação em função do grupo de competição de atletismo de Maringá, Paraná (2019).

\begin{tabular}{lccc}
\hline \multirow{2}{*}{ Estilos Regulatórios } & $\begin{array}{c}\text { Pista } \\
(\mathbf{n = 1 2})\end{array}$ & $\begin{array}{c}\text { Campo } \\
(\mathrm{n}=18)\end{array}$ & \\
\cline { 2 - 3 } & Md (Q1; Q3) & Md (Q1; Q3) & p \\
\hline Regulação Intrínseca & $7,00(7,00 ; 7,00)$ & $7,00(7,00 ; 7,00)$ & 0,74 \\
Regulação Integrada & $7,00(6,67 ; 7,00)$ & $6,67(6,17 ; 7,00)$ & 0,53 \\
Regulação Identificada & $6,67(6,00 ; 7,00)$ & $7,00(7,00 ; 7,00)$ & 0,17 \\
Regulação Introjetada & $7,00(6,83 ; 7,00)$ & $7,00(7,00 ; 7,00)$ & 0,66 \\
Regulação Externa & $3,00(2,66 ; 4,50)$ & $5,00(4,16 ; 5,00)$ & $0,03 *$ \\
Desmotivação & $1,33(1,00 ; 2,67)$ & $1,67(1,00 ; 2,33)$ & 0,96 \\
\hline
\end{tabular}

* Probabilidade estimada pelo teste de U de Mann-Whitney.

Padilha, Toigo e Saldanha (2020) e Minuzzi (2015) ressaltam que quanto mais autodeterminado maior o engajamento e interesse em atividades educacionais e de aprendizagem, causando uma maior permanência, empenho, entusiasmo e consequentemente uma atuação em longo prazo desses alunos nas atividades (exercícios) esportivas. Ademais, Decy e Ryan (1985) apontam que a ausência da motivação intrínseca e extrínseca resulta no sentimento de desmotivação, uma vez que o aluno/atleta não vê motivo (razão) para seguir no esporte, o que pode ocasionar em uma baixa frequência e/ou uma presença (atuação) somente por obrigação.

O fato das atletas, do sexo feminino, apresentarem maiores índices nos estilos regulatórios externos da mo- 
tivação pode demonstrar que as mulheres regulam sua motivação mais por fatores externos, como premiações materiais e financeiras, medo de punições, entre outros, do que quando comparado ao sexo masculino. Segundo Mizoguchi et al. (2014, p. 8) no momento em que as "pessoas estão focadas em metas extrínsecas, tais como em metas de desempenho, elas tendem a serem mais orientadas em direção a comparações interpessoais e sinais externos adquiridos de auto mérito". Portanto, nota-se que apesar de ambos os sexos se apresentarem motivados intrinsecamente, as mulheres apresentam influência por fatores ambientais (externos), o que reforça os achados de que em geral os homens são mais motivados em aspectos referenciados a competência esportiva, em especial na faixa dos 11 aos 14 anos (GAYA; CARDOSO, 1998).

Na comparação dos estilos regulatórios da motivação em função do grupo de competição de atletismo (provas de campo e de pista) ressalta-se que há poucos estudos em relação a este comparativo. Silva (2013) analisou que os atletas que se dedicam e empenham-se em treinamentos de longa duração, como o caso das provas de campo, por várias sessões durante o dia e por longas temporadas, muitas vezes não apresentam motivações intrínsecas suficientes para se manter no esporte, deste modo, a motivação extrínseca auxilia no envolvimento do aluno/atleta na tarefa (modalidade), driblando fatores como a exaustão, exigência de nível técnico e desgaste devido a rotina rígida (SILVA, 2018). Entretanto, conforme pôde ser observado, ainda que com provas distintas, os resultados motivacionais apresentaram-se com valores aproximados, que nos sugerem considerar a importância do ambiente e do instrutor como mediador da tarefa.

Ainda que com resultados mais próximos, se observarmos os atletas fundistas, podemos levantar outra hipótese, que se refere à liberação de endorfina, substância que promove redução da sensação de dores, favorecendo a melhora do humor e estimulando as sensações de bem-estar, o que se reflete nos aspectos emocionais do indivíduo e, em exercícios prolongados de base aeróbia a atividade cerebral das endorfinas são favorecidas (NAHAS, 2003; PEREIRA JÚNIOR; DE MIRANDA ROHLFS; LIMA, 2009), o que pode se refletir em uma maior motivação intrínseca para este grupo.

Já os autores Weinberg e Gould (2016) apontam como um fator para a motivação extrínseca o fato de o atleta possuir bolsa quando a bolsa assume o aspecto controlador, caracterizada por cobrança, punições ao atleta, como realização de tarefas sacrificantes, a mesma pode influenciar negativamente. Afinal, há uma relação para além do gosto pela modalidade e por competir, ou elementos relacionados ao comprometimento com o grupo. Quando existe o investimento, outros aspectos devem ser relacionados ao compromisso assumido, que se torna um apoio financeiro que, muitas vezes, traz consigo uma regulação externa no tocante à motivação de permanecer no esporte.

Frente aos resultados apresentados, a expectativa é que os treinadores e/ou professores atentem-se para os assuntos relacionados à motivação de seus alunos e/ou atletas. Desta maneira, esses profissionais precisam estabelecer estratégias e intervenções no dia a dia (cotidiano) de treinamento para que sempre haja bons motivos para a permanência no esporte.
Ressalta-se que o presente estudo se limita uma vez que retrata a realidade de um único grupo. Neste sentido, novos estudos podem ser realizados ampliando o tamanho da amostra e equiparando o número de atletas por tipo de prova, idade, nível técnico dos atletas, assim como a análise de acordo com o grupo de competição.

Do ponto de vista prática, destaca-se a importância de programas de treinamento que favoreçam o desenvolvimento da motivação autodeterminada e de que treinadores fiquem atentos para comportamentos regulados essencialmente por fatores externos, visto que tais comportamentos podem levar ao abando no da prática esportiva a longo prazo.

\section{CONCLUSÃO}

Concluiu-se que o sexo e o tipo de prova são fatores intervenientes na motivação extrínseca e desmotivação dos atletas de atletismo. Ressalta-se que as mulheres e os atletas de provas de campo apresentam comportamentos mais regulados por fatores como reconhecimento social e recompensas para a prática do atletismo.

\section{AGRADECIMENTOS}

Os autores agradecem a todos os atletas que aceitaram participar da pesquisa.

\section{CONFLITO DE INTERESSE}

Os autores do estudo declaram não haver conflito de interesses.

\section{FINANCIAMENTO}

Este estudo não teve apoio financeiro.

\section{REFERÊNCIAS}

ANDRADE, A. D. B.; COUTINHO, N. F. Atletismo na escola: é possível? Disponível em: <https://www.unaerp.br/revista-cientifica-integrada/edicoes-anteriores/volume-2-edicao-4/2053-atletismo-na-escola-e-possivel/file>. Acessado em: 15 de junho de 2021.

BENTO, A. N. F.; SILVA, M. R.; PONTES, M. Motivação de atletas federados para a prática de atletismo. Revista da Faculdade de Ciências da Saúde, Porto, v. 5, p. 326-36, 2008. Disponível em: <https://bdigital.ufp.pt/bitstream/10284/965/1/326-336.pdf>. Acessado em: 18 de fevereiro de 2022.

CONFEDERAÇÃO BRASILEIRA DE ATLETISMO. Histórico e provas do atletismo. Disponível em: <http://www.cbat.org.br/default.asp>. Acessado em: 15 de junho de 2021.

DECY, E. L.; RYAN, R. M. Intrinsic motivation and self-determination in human behavior. New York: Plenum, 1985.

DECY, E. L.; RYAN, R. M. The "whats" and "why" of goal pursuits: Human needs and the self determination. Psychological Inquiry, London, v. 11, n. 4, p. 227-68, 2009. DOI: https://doi.org/10.1207/S15327965PLI1104_01.

GAYA, A.; CARDOSO, M. Os fatores motivacionais para a prática desportiva e suas relações com sexo, idade e níveis de desempenho desportivo. Revista Perfil, Porto Alegre, v. 2, n. 2, p. 40-52, 1998. Disponivel em: < https:// seer.ufrgs.br/perfil/article/view/77333/pdf>. Acessado em: 18 de fevereiro de 2022

MINUZZI, T.; PANDOLFO, K. C. M.; AZAMBUJA, C. R.; MACHADO, R. R.; SANTOS, D. L. dos. Motivação de atletas escolares dos VII Jogos da Amizade: um estudo com o colégio militar de Santa Maria, RS. Cinergis, Santa Cruz do Sul, v. 16, n. 2, p. 1-4, 2015. DOI: https://doi.org/10.17058/cinergis.v16i2.6356. 
MIZOGUCHI, M. V.; GARCIA, W. F.; NAZARIO, P. F.; LARA, B. M. Análise dos níveis motivacionais dos corredores de rua de Bragança Paulista. Revista Brasileira de Psicologia do Esporte, Brasília, v. 7, n. 1, p. 1-11, 2014. DOI: http://dx.doi.org/10.31501/rbpe.v6i1.6720.

NAHAS, M. V. Atividade física, saúde e qualidade de vida: conceitos e sugestões para um estilo de vida ativo. 3. ed. Londrina: Madiograf, 2003.

NASCIMENTO JUNIOR, J. R. A.; VISSOCI, J. R. N.; BALBIM, G. M.; MOREIRA, C. R.; PELLETIER, L.; VIEIRA, L. F. Adaptação transcultural e análise das propriedades psicométricas da Sport Motivation Scale - II no contexto brasileiro. Revista da Educação Física, Maringá, v. 25, n. 3, p. 441-58, 2014. DOI: https://doi.org/10.4025/reveducfis.v25i3.24855.

PADILHA, L. D.; TOIGO, A. M.; SALDANHA, R. P. Análise dos estilos regulatórios da autodeterminação para a prática de atividades físicas e esportes em escolares. Revista Brasileira de Psicologia do Esporte, Brasília, v. 10, n. 1, p. p. 48-61, 2020. DOI: http://dx.doi.org/10.31501/rbpe.v10i1.11381.

PEREIRA JÚNIOR, A. A.; ROHLFS, I. C. P. M.; LIMA, W. C. Dependência ao exercício físico e a síndrome do excesso de treinamento: consequências do excesso de exercício. Revista da Unifebe, Brusque, v. 11, p. 1-9, 200. Disponível em: <http://docplayer.com.br/9054584-Dependencia-ao-exercicio-fisico-e-a-sindrome-do-excesso-de-treinamento-consequencias-do-excesso-de-exercicio.html>. Acessado em: 18 de junho de 2022

SAMULSKI, D. Psicologia do esporte. São Paulo: Manole, 2002.

SILVA, E. M.; MOURA, S. K. M. S. F.; RABAY, A. A. N.; FERREIRA, A. P. U.; SANTOS, R. M. L. Motivos de prática e abandono em corredores recracionais sob o ponto de vista da psicologia socialcognitiva. Diálogos em Saúde, Cabedelo, v. 1, n. 1, p. 80-92, 2019. Disponível em: <https://periodicos.iesp. edu.br/index.php/dialogosemsaude/article/view/201> Acessado em: 18 de fevereiro de 2022

SILVA, P. N.; SOLERA, B.; ALMEIDA, T. L.; ANVERSA, A. L. B.; MANOEL, F. A. Motivação para a prática da corrida de rua: diferença entre atletas de performance e atletas amadores. Caderno de Educação Física e Esporte, Marechal Cândido Rondon, v. 17, n. 2, p. 21-7, 2019. DOI: https://doi. org/10.36453/2318-5104.2019.v17.n2.p21.
SILVA M V da. Estado mental flow e motivação no atletismo: dos velocistas aos ultramaratonistas. 2013. 67f. Dissertação (Mestrado em Educação Física) - Universidade Federalde Juiz de Fora, Juiz de Fora, 2013.

SOUZA, R. S.; LIMA, J. R. P.; WERNECK, F. Z.; BARA FILHO, M. G.; FREITAS, V. Motivação de jovens e adultos para o treinamento do atletismo. Pensar a Prática, Goiânia, v. 19, n. 4, p. 914-25, 2016. DOI: https://doi.org/10.5216/ rpp.v19i4.40530.

WEINBERG, R. S.; GOULD, D. Fundamentos da psicologia do esporte e do exercício. 6. ed. Porto Alegre: Artmed Editora, 2016.

\section{ORCID E E-MAIL DOS AUTORES}

Giovana Correia da Silva

(iD) https://orcid.org/0000-0002-0801-2239

@ gcorreia17@gmail.com

Luciana Boligon Refundini

(iD https://orcid.org/0000-0003-3664-9402

Q luboligon@hotmail.com

Daniel Vicentini de Oliveira (Autor Correspondente)

(iD) https://orcid.org/0000-0002-0272-9773

@ d.vicentini@hotmail.com

Paula Caroline Teixeira Marroni

(iD) https://orcid.org/0000-0002-6831-3391

Q2paulamarroni@gmail.com

José Roberto Andrade do Nascimento Júnior

(iD https://orcid.org/0000-0003-3836-6967

Q jroberto.jrs01@gmail.com

Ana Luiza Barbosa Anversa

(iD https://orcid.org/0000-0003-4363-3433

@ana.beah@gmail.com 